\title{
Forum
}

\section{«Infant mental health» im deutschsprachigen Raum}

\section{Fernanda Pedrina (Zürich)}

Anfangs der 80er Jahren des letzten Jahrhunderts haben Psychotherapeuten begonnen, Säuglinge und Kleinstkinder als Subjekte in ihre Interventionen einzubeziehen; sie haben - nach dem Vorbild einzelner Pioniere - es gewagt, sich mit den jungen Patienten jenseits der sprachlichen Verständigung einzulassen. Seither sind auf Grund wachsender Erfahrungen und der zunehmenden Kenntnisse über Entwicklungsprozesse verschiedene Behandlungsmethoden für in psychische Not geratene Familien mit Babys ausgearbeitet worden. Sie finden Anwendung in Kompetenzzentren für kleine Kinder, die auch Psychotherapie anbieten oder in psychotherapeutischen «Babyambulanzen», die inzwischen gegründet worden sind und derzeit in vielen Städten am Entstehen oder geplant sind. Ziel dieser ForumsMitteilung ist es, diese Entwicklung im deutschsprachigen Raum nachzuzeichnen und die Bedeutung des psychoanalytischen Standpunktes in der fachlichen und institutionellen Debatte darzustellen.

\section{Eltern-Baby-Therapie: eine neue Option}

Eltern-Baby-Therapien sind die wichtigste Option und die erste Wahl unter den Behandlungsmöglichkeiten für die frühe Kindheit. Die Grundlagen für diese Intervention sind gleichzeitig in verschiedenen Disziplinen herangereift, insbesondere in der objekttheoretisch orientierten Psychoanalyse und in der akademischen Interaktionsforschung. Eine wichtige frühe Referenz auf psychoanalytischer Seite ist das Buch «Clinical studies in infant mental health» von Selma Fraiberg (1980). Darin hat diese Autorin - unter vielen anderen wegweisenden Aussagen - die Figur der (unbewussten) Projektion rigider, aus früheren Beziehungserfahrungen stammenden Vorstellungen der Eltern auf das Kind und ihren Einfluss auf die Bildung der kindlichen Symptomatik so überzeugend dargestellt, dass auch Fachpersonen im Frühbereich, die dem psychoanalytischen Denken abgeneigt sind, die Tatsache der transgenerationalen Weitergabe von Konflikten anerkennen. "Ghosts in the nursery» (Gespenster in der Kinderstube) ist die populär gewordene Bezeichnung für diese ungebetenen Gäste aus der Vergangenheit, die sich in der Eltern-KindBeziehung in Szene setzen. 
In dieser Anfangszeit war die interdisziplinäre Diskussion unter den zahlreichen Fachpersonen, die sich in Forschung und Klinik mit Babys befassten, sehr lebendig. Psychotherapeuten wurden durch die Vorführung von Forschungsvideos, die zum Teil in verlangsamtem Tempo gezeigt wurden, für die phänomenologischen Aspekte des averbalen Austausches zwischen Kind und Bezugsperson und auch für die differenzierte Erfassung der körperlichen Zeichen von Stress und Unbehagen beim Kind sensibilisiert.

Es sind verschiedene Formen der Eltern-Baby-Therapie entstanden, die unterschiedliche Mittel einsetzen (viele arbeiten videogestützt) und sich in der Fokuswahl unterscheiden. Die unterschiedliche disziplinäre Herkunft der Therapeuten (aus der Kommunikationsforschung, aus der Psychotherapie, aus der Sozialarbeit oder Frühpädagogik) beeinflusst zum Teil die bevorzugten Indikationsbereiche. Es sind jedoch immer mehr Überschneidungen und gegenseitige Anleihen erkennbar. Allen gemeinsam ist die Beachtung des Grundsatzes, dass die kindliche Entwicklung nur im Kontext von vertrauten Beziehungen gelingen kann.

So richtet sich das Interesse der Psychotherapeuten auf mindestens drei Prozesse:

, die Entwicklung der Beziehung zwischen Kind und Eltern (oder den bedeutendsten Betreuungspersonen), insbesondere unter dem Aspekt der Bindung,

, die psychische Strukturbildung beim Kind als Niederschlag von gelebten Interaktionen und unter dem Einfluss der beginnenden intrapsychischen Dynamik

, und die Elternschaftsentwicklung als Teil der Identitätsentwicklung bei Mutter und Vater, mit den vom Kind induzierten Veränderungen in allen wichtigen Lebensbereichen.

Das Phänomen der negativen Gegenseitigkeit, des schnellen Aufschaukelns von Unruhe und Verunsicherung in der frühen Interaktion zwischen Mutter und Säugling, macht, dass auch relativ gesunde Dyaden in Krisenzustände geraten können. Diese sind symptomatisch von schweren Dekompensierungen mit tiefgreifendem Störungshintergrund kaum zu unterscheiden. In leichteren Fällen mit funktioneller Symptomatik kann die Fokussierung der therapeutischen Intervention auf die Interaktion genügen. Bei schweren Störungen und mit zunehmender Dauer der Belastung entwickelt das Kind ein stabileres psychopathologisches Bild, das einen umfassenderen Zugang erfordert. In den ersten kommunikationsorientierten Babyambulanzen im deutschsprachigen Raum erhielten 
so genannte Regulationsstörungen (Schlaf- und Essverhaltensstörungen sowie exzessives Schreien) einen zentralen Stellenwert, der durch die Erstellung einer AWMF-Leitlinie untermauert wurde. Es ist der Verdienst psychoanalytischer Therapeuten, dass auch andere frühe psychische und psychosomatische Störungen in den Blick genommen wurden. Mit der Verfassung einer eigenen, breiter gefassten Leitlinie haben sie eine diagnostische Vorgehensweise skizziert, welche die Erfassung relevanter intrapsychischer Aspekte der Interaktionspartner miteinbezieht und dementsprechend gezielte, psychoanalytisch begründete Interventionen zur Diskussion stellt (Berger et al. 2006).

Die Beschreibung psychischer Störungen im frühen Kindesalter ist inzwischen soweit differenziert worden, dass diese in einem eigenen Klassifikationssystem zusammengestellt sind (ZTT, Zero to Three Diagnostic Classification, 2005). Die Krankheitsbilder der Kinder werden dabei gesondert von den ebenfalls klassifizierten Beziehungsstörungen aufgeführt. Der ZTT wird derzeit in einem internationalen Konsensverfahren in Hinblick auf die Integration in die allgemeine Klassifikation psychischer Störungen weiterentwickelt.

\section{Vom Baby im Kinderzimmer zum Baby in der Gesellschaft}

Die Verbreitung der Eltern-Baby-Therapie ist möglicherweise durch die Zuversicht unterstützt worden, die die Pioniere in diesem Gebiet geäussert hatten. «You have God on your side», hatte Fraiberg im bereits erwähnten Buch geschrieben. Psychotherapeuten konnten mit Freude erfahren, dass die positive Gegenseitigkeit in frühen Interaktionen ebenso wirksam Genesungsprozesse unterstützt, wie die negative zur Entstehung von Symptomen führt. Mit zunehmender Erfahrung kam aber auch die Konfrontation mit schweren Störungen, die sich nicht so leicht beheben lassen. Auf der Seite des Kindes erwähne ich posttraumatische Zustände, wie zum Beispiel die Fütterstörung, die sich unter Umständen nach lang dauernder künstlicher Ernährung bei einem Frühgeborenen einstellt und uns vor grosse Probleme bei der Sondenentwöhnung stellt. Auf Seiten der Eltern sind psychische Krankheiten, die sie in ihrer elterlichen Kompetenz beeinträchtigen, zunehmend ins Blickfeld geraten.

Schon früh wurde die postpartale Depression, mit ihren nachteiligen Auswirkungen auf die Mutter-Kind-Interaktion und auf die kindliche Entwicklung thematisiert. Eine längere Tradition hat die zuerst in England erprobte gemeinsame psychiatrische Hospitalisation von Mutter und Baby in schwersten Fällen, insbesondere bei der postpartalen Psychose. Die Absicht der behandelnden Ärzte ist dabei, die längerfristige Eignung der psychotischen Mutter abzuklären, unter 
Behandlung und in einem haltenden Umfeld ihr Kind zu betreuen. Durch die Vermeidung der frühen Trennung wäre die Entwicklung der Bindung nicht präjudiziert. Bei der ambulanten Behandlung der Depression hat sich mit der Zeit gezeigt, dass der therapeutische Erfolg nicht so sehr vom Schweregrad der depressiven Symptomatik abhängig ist, sondern vielmehr von der psychischen Struktur der betroffenen Mutter. So kann aus einer Eltern-Kind-Intervention bei einer relativ banalen Symptomatik (z. B. Schlafstörung des Kindes, Erschöpfung der Mutter, Paarstreitereien) eine längere Therapie entstehen, wenn es sich herausstellt, dass die Mutter in ihrem Leben allgemein grosse Mühe hat, ihre Beziehungen zu gestalten sowie ihre Stimmungen und ihr Selbstwertgefühl zu regulieren. «BorderlineMütter» und die Probleme, die sich bei der Betreuung ihrer Kinder konstellieren, stehen heute im Zentrum der Sorge vieler Fachleute im Bereich der frühen Kindheit. $\mathrm{Zu}$ diesem Thema hat die psychoanalytische Forschung in den letzten Jahren viele Erkenntnisse und Modelle erarbeitet. Einerseits sind genauere Zusammenhänge zwischen der Selbstentwicklung des Kindes und der Qualität der Affekspiegelung, die im vertrauten Austausch des Kindes mit seinen engen Bezugspersonen stattfindet, beschrieben sowie Bezüge zu späteren Persönlichkeitsentwicklungsstörungen hergestellt worden (Fonagy et al. 2002). Andererseits hat die therapeutische Arbeit mit Patienten mit Persönlichkeitsstörungen deutlich gemacht, wie wichtig es ist, die Integrierung der psychischen Struktur zu berücksichtigen und zu stützen, bevor die vorliegenden Konflikte ohne die Gefahr einer zusätzlichen Destabilisierung bearbeitet werden können (Rudolf 2006).

Die psychoanalytische Sichtweise hat sehr viel zum Verständnis der frühen Beziehung eines Patienten zu seiner als persönlichkeitsgestört erkannten Mutter beigetragen. Bisher fanden diese Kinder erst spät (manchmal erst nach dem Ableben der Mutter) aus eigener Kraft den Weg zur Psychotherapie. In einer neueren Arbeit wurden aus zahlreichen Behandlungen retrospektiv typische Erziehungshaltungen, Konflikte, destruktive Verwicklungen ausgearbeitet, die zu früheren Formen der Hilfe motivieren und die Formulierung therapeutischer Hypothesen für die Interventionen im Mutter-Kind-Setting unterstützen (Lawson 2000). Die gleiche Autorengruppe, die die Leitlinien zu den psychischen Störungen im Säuglingsalter erstellt hatte, hat später ein Therapiemanual erarbeitet, in dem die neuen Erkenntnisse in Bezug auf den Umgang mit persönlichkeitsgestörten Eltern eingeflossen sind. Neben den allgemeinen Anweisungen zur Herstellung eines therapeutischen Milieus, in dem die Eltern zurWahrnehmung der averbalen Mitteilungen des Kindes sensibilisiert und zur bewussten Reflexion der Erfahrung mit ihm unterstützt werden, werden spezifische Interventionen für einen eher 
konfliktbezogenen oder für einen eher strukturbezogenen Behandlungsfokus bei den Eltern empfohlen (Cierpka und Windhaus 2007: 216). Andere Eltern-SäuglingTherapeuten, die vorwiegend interaktionszentriert arbeiten, überweisen in solchen Fällen die Mutter oder die Eltern dem Erwachsenenpsychiater. Da die Behandlung einer Persönlichkeitsstörung aber meistens langwierig ist, darf das Kind nicht aus den Augen verloren gehen: seine Entwicklung ist aktuell in Gefahr, es kann nicht solange warten, bis die Eltern stabilisiert sind.

Noch wichtiger als die Kompetenz der psychoanalytischen Psychotherapeuten im Umgang mit elterlichen Pathologien ist aber in schweren Fällen ihre Fähigkeit, in einem multiprofessionellen Netzwerk zu funktionieren. Bei schwer dekompensierten Familien mit Babys sind Entlastungs- und Unterstützungsmassnahmen, unter Umständen Stunden oder Tage mit extrafamiliärer Betreuung nötig. Manchmal sind die Eltern nicht genügend gewahr, wie sehr das Kind leidet oder sie sind nicht in der Lage, das Nötige zu tun, um seine Not zu mildern: dann müssen Aussenstehende elterliche Funktionen übernehmen. Eltern müssen überzeugt werden, dass sie Unterstützung annehmen müssen, und wenn es nicht gelingt müssen sie durch Meldung an die Behörde dazu gezwungen werden. Es ist vielen Kollegen noch nicht bekannt, dass das Gesetz im Bereich der frühen Kindheit einen präventiven Kinderschutz vorsieht. Die Vormundschaftsbehörde (in der Schweiz) ist wie der Psychotherapeut primär bestrebt, Entwicklungskrisen zu beheben, Entwicklungsprozesse aufzugleisen und wenn möglich das Kind in seiner Familie zu belassen. Mit diesem Hintergrund ist Zusammenarbeit sinnvoll, die Eltern-Kind-Therapie kann ein wertvolles Element in einem übergeordneten, von der Behörde (d.h. in der Regel von einem fachkundigen Sozialarbeiter) erarbeiteten und gehaltenen Unterstützungssystem sein. Im Bereich der frühen Hilfen weist die Schweiz innerhalb des deutschsprachigen Raumes eine privilegierte Situation auf. Seit Jahrzehnten ist bei uns die Institution der Mütterberatung als eine für alle verfügbare, kostenlose, psychologisch und materiell niederschwellige Anlaufstelle etabliert. Familienkrisen und postpartale Depressionen werden dort viel früher gemeldet als in ärztlichen Praxen. Ebenso verbreitet und akzeptiert sind bei uns Kinderkrippen, wodurch schon seit langem nicht mehr über ihre Existenzberechtigung sondern vielmehr über deren Qualität diskutiert wird. In diesem Kontext hat eine interdisziplinäre Projektgruppe in Zürich unter der Schirmherrschaft der GAIMH (deutschsprachige Gesellschaft für die seelische Gesundheit in der frühen Kindheit) Richtlinien für den Kinderschutz in der frühen Kindheit - 0 bis 3 Jahre - erarbeitet (Mahrer, Meier, Mögel, Pedrina, Ryf, Simoni, 2007). Diese gehen von den besonderen Entwicklungsbedürfnissen kleiner 
Kinder aus, stellen die Belastungssymptomatik sowie die spezifischen sozialen Hilfestellungen für Familien mit kleinen Kindern dar, erläutern das Vorgehen über die Behörde im Falle der Gefährdung und zeigen Szenarien der vernetzten therapeutischen Arbeit bei freiwilliger Kooperation seitens der Eltern oder im gesetzlich festgelegten Rahmen auf. Die Broschüre, an deren Konzipierung psychoanalytische Therapeutinnen massgeblich beteiligt waren, ist bereits in 3. Auflage erschienen und findet grossen Anklang in vielen Sozialdiensten und Vormundschaftsstellen. Es zeichnet sich ab, dass ein lebendiges Netz von Fachleuten der frühen Kindheit entstehen könnte, die an einer gemeinsamen Kultur in Bezug auf die Entwicklung kleiner Kinder in deren familiären und sozialen Kontext teilhaben. Ein solcher informeller Konsens bedeutet eine unschätzbare Hilfe für die einzelnen Unterstützungsbemühungen. An klinischen Fallgeschichten (wofür hier leider kein Platz ist) könnte ich aufzeigen, wie schwer belastete Babys mit ihren Eltern durch vernetzte, gesetzlich vorgeschriebene Begleitungen, inkl. Psychotherapie, doch noch ohne traumatische Fremdplatzierungen aus der Krise fanden. Oder auch wie ein schwer bedrohtes Kind (oder im Falle eines Münchhausen by-proxySyndrom) vor der verschlingenden-destruktiven Betreuung seiner Mutter durch die Heimplatzierung auf die Dauer bewahrt werden konnte, ohne den Kontakt zu seiner Mutter zu verlieren.

\section{Institutionelle Entwicklungen in der deutschsprachigen Schweiz}

Wegen der engen Verflechtung von entwicklungsfördernden und therapeutischen Aufgaben im Bereich der frühen Kindheit sei ein Hinweis auf die Situation über die Fachgrenzen hinweg gestattet. In den letzten Jahrzehnten haben sich Angebote vervielfacht, die sich an Eltern mit kleinen Kindern im Bereich der Entwicklungsbegleitung und -beratung wenden. Familienergänzende Angebote sowie die Kindsbetreuung in Kinderheimen werden durch die Verbreitung frühpädagogischen Wissens professionalisiert. Im therapeutischen Bereich werden Eltern-Kind-Therapie in einigen kinderpsychiatrischen Diensten integriert, nachdem sie längere Zeit von einzelnen ambulant tätigen Psychotherapeuten geleistet wurden. Entwicklungspädiatrische Zentren ergänzen zum Teil ihre Praxis der Entwicklungsberatung mit psychotherapeutischen Elementen. Es sind Möglichkeiten der gemeinsamen Unterbringung von Mutter und Baby in postpartalen Krisen in mehreren Institutionen (in Kinder- und Jugendheimen, in pädiatrischen oder psychiatrischen Abteilungen, im allgemeinen Krankenhaus) entstanden. Das Umfeld für die frühen Hilfen für Familien mit kleinen Kindern 
ist in Bewegung, das Bedürfnis seitens betroffener Familien ist jedoch in vieler Hinsicht noch nicht abgedeckt.

Für Psychotherapeuten, die sich die Kompetenz erarbeiten wollen, ElternBaby/Kleinkind-Therapien durchzuführen, stehen heute einige postgraduale Ausbildungscurricula zurVerfügung. Eine deutliche psychoanalytische Orientierung weisen das Curriculum der Fachhochschule Potsdam-Berlin (Leitung Prof. C. Ludwig-Körner) sowie dasjenige der ärztlichen Akademie für Psychotherapie von Kindern und Jugendlichen in München (Leitung Dr. med. M. Endres) auf. Beide setzen eine abgeschlossene Psychotherapieausbildung voraus.

\section{Literatur}

Berger, Margarete, Elfi Freiberger, Barbara von Kalchreuth, Maria Knott, Christiane Wiesler und Eberhard Windhaus (2006): Leitlinien Regulationsstörungen, psychische und psychosomatische Störungen im Säuglings- und frühen Kindesalter. In: Analytische Kinder- und Jugendlichenpsychotherapie, 37, 545-576.

Cierpka, Manfred and Eberhard Windhaus (2007): Psychoanalytische SäuglingsKleinkind-Eltern-Psychotherapie. Frankfurt a.M.: Brandes \& Apsel.

Fonagy, Peter, Gyorgy Gergely, Elliot L. Jurist and Mary Target (2002): Affect regulation, mentalization and the development of the self. New York: Other Press LLC.

Fraiberg, Selma (1980): Clinical studies in infant mental health. The first year of life. New York: Basic Books.

Lawson, Christine A. (2000): Understanding the borderline mother: helping her childern trascend the intense, unpredictable and volatile relationship. Northvale, NJ: Jason Aronson.

Mahrer, Monika, Peter Meier, Maria Mögel, Fernanda Pedrina, Esther Ryf und Heidi Simoni (2007): Kindesschutz in der frühen Kindheit 0-3 Jahre. Zürich: GAIMH, Gesellschaft für die seelische Gesundheit in der frühen Kindheit e.V., Interdisziplinäre Regionalgruppe Zürich.

Pedrina, Fernanda (1997): Intrapsychisches und interaktionelles Geschehen in den Säuglings-Eltern-Therapien. Arbeitshefte Kinderpsychoanalyse, 24, 119-133.

Pedrina, Fernanda (2006): Mütter und Babys in psychischen Krisen. Forschungsstudie zu einer therapeutisch geleiteten Mutter-Säugling-Gruppe am Beispiel postpartaler Depression. Frankfurt a.M.: Brandes \& Apsel. 
Rudolf, Gerd (2006): Strukturbezogene Psychotherapie: Leitfaden zur psychodynamischen Therapie struktureller Störungen. Schattauer. Stuttgart.

Zero to Three, Diagnostic Classification Task Force (2005): DC: 0-3 R, Diagnostic classification of mental health and developmental disorders of infancy and early childhood. Washington D. C.: Zero to Three Press.

\section{Anmerkungen}

1 Arbeitsgemeinschaft der Wissenschaftlichen Medizinischen Fachgesellschaften (www.awmf.org).

2 In meiner Zürcher Praxis werden Eltern-Baby-Therapien von mir und meinen Mitarbeitern seit mehr als 25 Jahren durchgeführt (Pedrina, 1997, 2006). 